\title{
Stacking of adjacent graphene layers grown on C-face SiC
}

Leif I Johansson, Somsakul Watcharinyanon, A A Zakharov, Tihomir Iakimov, Rositsa Yakimova and Chariya Virojanadara

\section{Linköping University Post Print}

N.B.: When citing this work, cite the original article.

Original Publication:

Leif I Johansson, Somsakul Watcharinyanon, A A Zakharov, Tihomir Iakimov, Rositsa Yakimova and Chariya Virojanadara, Stacking of adjacent graphene layers grown on C-face SiC, 2011, Physical Review B. Condensed Matter and Materials Physics, (84), 12, 125405.

http://dx.doi.org/10.1103/PhysRevB.84.125405

Copyright: American Physical Society http://www.aps.org/

Postprint available at: Linköping University Electronic Press http://urn.kb.se/resolve?urn=urn:nbn:se:liu:diva-70733 


\title{
Stacking of adjacent graphene layers grown on $\mathrm{C}$-face $\mathrm{SiC}$
}

\author{
L. I. Johansson, ${ }^{1, *}$ S. Watcharinyanon, ${ }^{1}$ A. A. Zakharov, ${ }^{2}$ T. Iakimov, ${ }^{1}$ R. Yakimova,,${ }^{1}$ and C. Virojanadara ${ }^{1}$ \\ ${ }^{1}$ Department of Physics, Chemistry and Biology, Linkoping University, S-58183 Linkoping, Sweden \\ ${ }^{2}$ MAX-lab, Lund University, S-22100 Lund, Sweden
}

(Received 21 June 2011; revised manuscript received 1 August 2011; published 6 September 2011)

\begin{abstract}
Graphene was grown on the $\mathrm{C}$-face of nominally on-axis $\mathrm{SiC}$ substrates using high-temperature sublimation with Ar as the buffer inert gas. The results of studies of the morphology, thickness, and electronic structure of these samples using low-energy electron microscopy (LEEM), x-ray photoelectron emission microscopy, photoelectron spectroscopy, angle-resolved photoelectron spectroscopy (ARPES), and low-energy electron diffraction (LEED) are presented. The graphene thickness is determined to vary from 1 or 2 to 6 or 7 monolayers (MLs), depending on the specific growth conditions utilized. The formation of fairly large grains (i.e., crystallographic domains) of graphene exhibiting sharp $1 \times 1$ spots in micro-LEED is revealed. Adjacent grains are found to show different azimuthal orientations. Macro-LEED patterns recorded mimic previously published, strongly modulated, diffraction ring LEED patterns, indicating contribution from several grains of different azimuthal orientations. We collected selected area constant initial energy photoelectron angular distribution patterns that show the same results. When utilizing a small aperture size, one Dirac cone centered on each of the six K-points in the Brillouin zone is clearly resolved. When using a larger aperture, several Dirac cones from differently oriented grains are detected. Our findings thus clearly show the existence of distinct graphene grains with different azimuthal orientations; they do not show adjacent graphene layers are rotationally disordered, as previously reported for $\mathrm{C}$-face graphene. The graphene grain size is shown to be different on the different samples. In some cases, a probing area of $400 \mathrm{~nm}$ is needed to detect the grains. On one sample, a probing area of $5 \mu \mathrm{m}$ can be used to collect a $1 \times 1$ LEED pattern from a multilayer graphene grain. ARPES is used to determine the position of the Dirac point relative to the Fermi level on two samples that LEEM shows have dominant coverage of 2 and 3 MLs of graphene, respectively. The Dirac point is found to be located within $75 \mathrm{meV}$ of the Fermi level on both samples, which indicates that the electron carrier concentration induced in the second and third graphene layers on the C-face is less than $\sim 4 \times 10^{11} \mathrm{~cm}^{-2}$. Formation of patches of silicate is revealed on some samples, but the graphene formed on such nonhomogenous surfaces can contain fairly large ordered multilayer graphene grains.
\end{abstract}

DOI: 10.1103/PhysRevB.84.125405

PACS number(s): 68.65.Pq, 73.22.Pr, 81.05.ue, 68.37.Nq

\section{INTRODUCTION}

Epitaxial graphene grown on the C-terminated $\mathrm{SiC}(000-1)$ surface has been found to be dramatically different ${ }^{1-7}$ compared to graphene grown on the Si-terminated $\mathrm{SiC}(0001)$ surface. A rotational disorder between adjacent graphene layers has been reported for $\mathrm{C}$-face graphene. Whereas $\mathrm{Si}$ face graphene exhibits sharp low-energy electron diffraction (LEED) spots and Bernal (ABAB...) stacking, the LEED diffraction pattern obtained from $\mathrm{C}$-face graphene is smeared out into a strongly modulated diffraction ring. 2,4,5,7 This, together with surface $\mathrm{x}$-ray diffraction results, ${ }^{1,2,5}$ was interpreted to indicate that the graphene layers on the $\mathrm{C}$-face stack in such a way that adjacent layers are rotated with respect to each other. The experimental results indicated some preferred rotation angles, but a turbostratic structure ${ }^{8}$ was proposed $^{2,4}$ for multilayer graphene films on the $\mathrm{C}$-face. Moreover, the rotated layers were suggested to be interleaved, rather than forming distinct domains of different orientations. The rotational disorder produces moiré patterns in scanning tunneling microscopy (STM) topographs ${ }^{6}$ and has been suggested to explain why epitaxial graphene films, tens of layers thick, show single-layer electronic properties, ${ }^{2,5}$ i.e., show a single $\pi$-band cone with the Dirac point at the Fermi energy. For the Si-face, the first carbon layer formed, the so-called buffer layer, is known to form strong covalent bonds to the $\mathrm{SiC}$ substrate, so this carbon layer does not exhibit the electronic properties of graphene. It shows no graphene $\pi$-band at the K-point in the Brillouin zone ${ }^{4}$ and the $\mathrm{C}$ 1s core level from this layer exhibits two components $^{4,9}$ instead of one graphene/graphite peak. The first carbon layer developing on the $\mathrm{C}$-face gives rise to a single $\mathrm{C} 1 \mathrm{~s}$ peak $^{4}$ and no interface-related component, which indicates an absence of strong covalent bonds to the substrate. A theoretical mode ${ }^{10}$ of the graphene C-face interface, however, suggested the presence of strong covalent bonds and thus the existence of a buffer carbon layer on the C-face. A recent study ${ }^{11}$ of the substrate-induced charge density profile in $\mathrm{C}$-face epitaxial graphene determined the Fermi level in the first three heavily doped graphene layers to be located $360-140 \mathrm{meV}$ above the Dirac point. If this is the case, for a graphene film 2-3 monolayers (MLs) thick, we would expect to be able to reveal that the Dirac point is located far below the Fermi level, like in the case of monolayer graphene on the Si-face. ${ }^{12}$

For Si-face graphene, the structural quality has been shown to improve dramatically upon furnace growth at a higher temperature and in an argon ${ }^{13,14}$ or disilane ${ }^{15}$ environment. A recent study of $\mathrm{C}$-face graphene did not show similar improvement ${ }^{7}$ after preparation in a 1-atm argon environment compared to in situ preparation. Instead of a layer-by-layer growth, like on the Si-face, the formation of graphene islands was revealed, and they had to grow relatively thick ( $\geqslant 5 \mathrm{MLs}$ ) samples before complete graphene coverage was achieved.

We prepared graphene films on the $\mathrm{C}$-face of $\mathrm{SiC}$ to a thickness varying from 1 or 2 to 6 or 7 MLs and investigated these samples using a number of surface science tools. What we found was formation of fairly large grains (crystallographic 
domains) of graphene exhibiting sharp $1 \times 1$ spots in microLEED (selected area LEED); adjacent grains were found to show different azimuthal orientations. Macro-LEED patterns collected were found to mimic earlier recorded $2,4,5,7$ and schematically drawn ${ }^{3}$ LEED patterns and indicated contribution from several grains of different azimuthal orientations. We collected selected area constant initial energy photoelectron angular distribution patterns that showed the same results. When utilizing a small aperture (probing area), the six Dirac cones at the K-points of the Brillouin zone could be clearly resolved, whereas several Dirac cone replicas from differently oriented grains were detected when a large aperture was utilized. These findings show the existence of distinct graphene grains with different azimuthal orientations; they do not show adjacent graphene layers are rotationally disordered, as previously reported for $\mathrm{C}$-face graphene. In addition, angle resolved photoemission data collected from samples with predominantly 2 and 3 MLs of graphene showed the Dirac point to be located very close to the Fermi level and not the 215 and $140 \mathrm{meV}$ below Fermi, as determined in a recent study $^{11}$ of a 63-layer-thick sample using infrared pump-probe spectroscopy. However, like another recent study of C-face graphene prepared in an Ar environment, ${ }^{7}$ pronounced island formation was revealed on some samples; in addition, patches of oxide (silicate) were detectable on the areas not covered by graphene.

\section{EXPERIMENTS}

Graphene was grown on nominally on-axis $\mathrm{SiC}$ substrates with the aim of obtaining graphene samples with a thickness from 1 or 2 to $\sim 10$ MLs. The substrate was, with one exception, production grade n-type $6 \mathrm{H}$ from SiCrystal, with a misorientation error within $0.06^{\circ}$, and was cleaned using the standard Radio Corporation of America (RCA) cleaning procedure before introduction into the sublimation furnace. One sample was prepared on an on-axis $4 \mathrm{H}$ substrate. Hightemperature sublimation with a buffer inert gas was used, ${ }^{13,16}$ and a temperature range of $1800-2000{ }^{\circ} \mathrm{C}$, pressure range of 500-850 mbar, and average growth time of 15 min were applied. The exact growth condition for each sample is given in the caption of Figs. 1 and 3 to 6 . We utilize a similar buffer gas pressure and growth time but a considerably higher temperature than the $\sim 1600{ }^{\circ} \mathrm{C}$ used in the most recently reported study. ${ }^{7}$ The thickness, morphology, and electronic structure of the graphene samples prepared were investigated using low-energy electron microscopy (LEEM), x-ray photoelectron emission microscopy (XPEEM), photoelectron spectroscopy (PES), and LEED on beam line I311 at the MAX Laboratory in Sweden. This beam line is equipped with a modified SX-700 monochromator, which provides light for two end stations. The first station is for PES. It is built around a large hemispherical Scienta electron analyzer and operates at a base pressure of $\sim 1 \times 10^{-10}$ mbar. A total energy resolution of $10-100 \mathrm{meV}$ at photon energies from 33 to $450 \mathrm{eV}$ and of $<300 \mathrm{meV}$ at energies from 600 to $750 \mathrm{eV}$ was selected for high-resolution studies of the core levels and the valence band. The second end station is built around a LEEM (LEEM III, Elmitec) instrument, which also allows for XPEEM studies. Linearly, s-polarized radiation of the first harmonics from an undulator is then used for the excitation at normal incident to the sample surface (the synchrotron light goes through the beam separator of the microscope). The energy-filtering function is realized by utilizing a hemispherical analyzer in the electron optical path. It is possible to switch between energyfiltered images of the surface and energy-filtered photoelectron emission angular distributions. In addition, angle-resolved photoelectron spectroscopy (ARPES) experiments were performed at beam line I4. This beam line is equipped with a spherical grating monochromator and a Phoibos 100 twodimensional charge-coupled device Specs energy analyzer. The low angular dispersion lens mode was selected, which provided an acceptant angle of $\pm 7^{\circ}$. In the ARPES and PES measurements, a Ta foil mounted on the sample manipulator was utilized as a reference sample for determining the Fermi level. The samples grown were load locked into these different instruments and then outgassed to $\sim 500{ }^{\circ} \mathrm{C}$ for a few minutes prior to taking measurements. Data collection was carried out with the sample at room temperature.

\section{RESULTS AND DISCUSSION}

A LEEM image collected at a voltage of $0.4 \mathrm{eV}$ and a field of view of $20 \mu \mathrm{m}$ is shown in Fig. 1(a). Several domains are clearly visible. To determine the number of layers present, the electron reflectivity versus electron kinetic energy, the so-called I-V curve, was extracted from the different domains. The curves obtained from areas A-D are displayed in Fig. 1(b). From the lightest gray areas, labeled A, the curve exhibits one minimum, which means ${ }^{17}$ that these areas have a graphene layer thickness of 1 ML. Most of the surface area in Fig. 1(a)
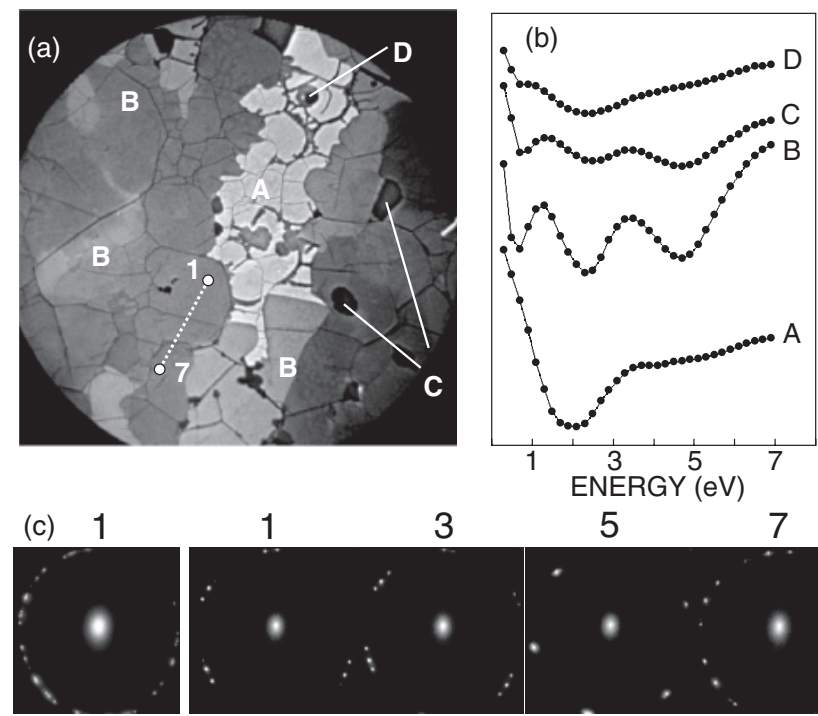

$5 \mu \mathrm{m}$

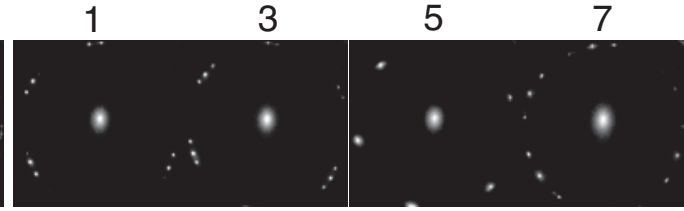

$0.4 \mu \mathrm{m}$

FIG. 1. (a) LEEM image recorded at $0.4 \mathrm{eV}$ and a field of view of $20 \mu \mathrm{m}$. (b) Electron reflectivity curves extracted from areas A-D. (c) A LEED pattern collected from the 3-ML graphene area at 44 $\mathrm{eV}$ using a probing area of $5 \mu \mathrm{m}$ is shown in the far left panel labeled 1 . The other four panels show LEED patterns collected at the same energy but using a probing area of $400 \mathrm{~nm}$ at four equidistant positions, from position 1 to position 7 in (a). This sample was grown at $1850{ }^{\circ} \mathrm{C}$ for $15 \mathrm{~min}$ in an $\mathrm{Ar}$ pressure of $500 \mathrm{mbar}$. 
appears light gray and correspond to areas B. Curve B shows three minima, so the graphene coverage is $3 \mathrm{MLs}$ on most parts of the surface. The darker gray patches, labeled $\mathrm{C}$ and enclosed by $\mathrm{B}$ areas, also have a graphene coverage of $3 \mathrm{MLs}$, whereas the patches labeled D and enclosed by A areas have a coverage of $1 \mathrm{ML}$. The graphene coverage is thus fairly homogenous for a C-face sample, ${ }^{7}$ because the domain size is on the order of micrometers, but is not as good as for Si-face samples, where domain sizes of tens of micrometers have been obtained. ${ }^{18,19}$ The domains we refer to are areas of constant graphene thickness that are observed in LEEM and XPEEM images. When we later write "grains," we refer to crystallographic domains, which are considerably smaller areas, as revealed later. An interesting observation is revealed in Fig. 1(c), where a set of selected area LEED diffraction patterns, collected at an electron energy of $44 \mathrm{eV}$, are shown. The pattern displayed in the panel at the left, labeled 1 and $5 \mu \mathrm{m}$, was collected from position 1 using a probing area of $5 \mu \mathrm{m}$. It resembles quite well the smeared out and strongly modulated ringlike diffraction pattern reported earlier. ${ }^{2,4}, 5,7$ When selecting a probing area of only $400 \mathrm{~nm}$, the LEED pattern from position 1 appears quite different, as seen in the second panel from the left. The diffraction pattern using this small aperture was collected at equidistant steps from position 1 to position 7 and included in Fig. 1(c), are the LEED patterns obtained at positions 3, 5, and 7. Clear and distinct variations in the LEED patterns are observed among the different positions, At position 5, a grain of the 3-ML graphene with one azimuthal orientation is probed. At this electron energy, LEED probes about four layers of graphene, as demonstrated ${ }^{13}$ for $\mathrm{Si}$-face graphene samples, where the LEED signal from the $6 \sqrt{3} \times 6 \sqrt{3} \mathrm{R} 30^{\circ}$ reconstructed carbon buffer layer is barely visible for a 3-ML graphene sample and is absent for a 4-ML sample. These observations are clearly different from what has been previously reported. In the recent comparative study ${ }^{7}$ of graphene grown on the C-face in situ and in an argon environment, similar selected-area LEED patterns using a probing area of $2 \mu \mathrm{m}$ were collected and analyzed. It was reported that the patterns at scores of locations over the surface, spaced $\sim 5 \mu \mathrm{m}$ apart, were examined and that no variation in the patterns were observable. The results implied $^{7}$ that either the crystallographic orientation did not change over the surface or that the grain size was much smaller than the $2-\mu \mathrm{m}$ sampling size utilized. Because the former was impossible, the conclusion was that, consistent with prior works, the grain size was much less than $2 \mu \mathrm{m}$. The micro-LEED patterns in Fig. 1(c) show that a grain size of $\sim 400 \mathrm{~nm}$ can be obtained and that adjacent graphene layers in those grains do not show any rotational disorder. Instead, the results imply the formation of ordered graphene grains with different azimuthal orientations. Before elaborating more about this, let us first look at the $C 1$ s core level and the $\pi$-band structure around the K-point in Brillouin zone for this sample.

C 1s spectra collected from this " 3 ML" graphene sample at three photon energies are shown in Fig. 2(a). They are found to be modeled accurately using only two components, the $\mathrm{SiC}$ component from the substrate and the $\mathrm{G}$ component from the graphene layers. In agreement with earlier results, ${ }^{4}$ no trace of a carbon buffer layer component can be observed on this surface. The energy separation of $1.9 \mathrm{eV}$ and peak intensity
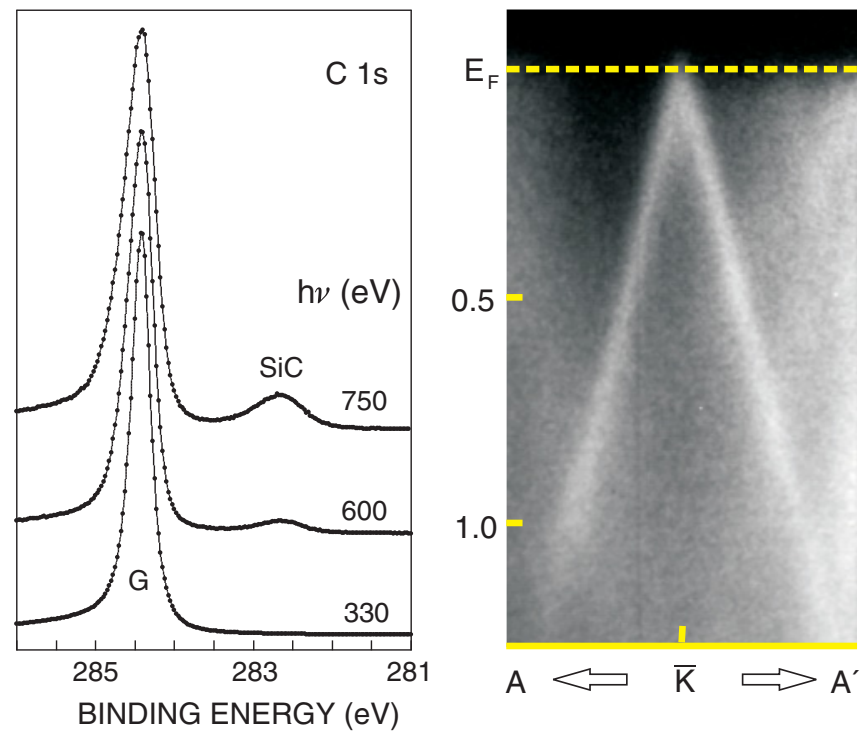

FIG. 2. (Color online) (a) Normal emission C 1s spectra recorded at three photon energies from sample 1, with mainly 3 MLs of graphene. (b) The $\pi$-band collected at the K-point from this sample. A photon energy of $33 \mathrm{eV}$ was used.

ratio of 23 (at $600 \mathrm{eV}$ ), determined between the graphene and the $\mathrm{SiC}$ components $(\mathrm{G} / \mathrm{SiC})$ are consistent with previously determined values ${ }^{4}$ for a graphene thickness of $\sim 3$ MLs.

Figure 2(b) shows the $\pi$-band recorded from this 3 -ML graphene sample around the K-point using a photon energy of $33 \mathrm{eV}$. The Dirac point is seen to be located very close to the Fermi level, within $50 \mathrm{meV}$, rather than at $140 \mathrm{meV}$ below the Fermi level, as determined recently ${ }^{11}$ using infrared pump-probe spectroscopy. Because the graphene grain size was only on the order of few hundred nanometers for this sample, a few $\pi$-cones were always detectable when moving the light spot over the sample. However, the Dirac point of the different observed cones was always located at the same energy position very close to the Fermi level. Because, for the second graphene layer, the Dirac point was determined ${ }^{11}$ to be located $215 \mathrm{meV}$ below the Fermi level, we searched for a 2-ML graphene sample so that we could check this using ARPES.

A LEEM image collected from a second sample at a voltage of $0.4 \mathrm{eV}$ and a field of view of $25 \mu \mathrm{m}$ is shown in Fig. 3(a). Extracted reflectivity curves from the areas labeled A and E-G are plotted in Fig. 3(b). The lightest gray areas, labeled $\mathrm{A}$, are again seen to correspond to areas of monolayer graphene, because curve A shows only one dip. The dominant gray areas labeled $\mathrm{E}$ and $\mathrm{F}$ are seen to represent areas with 2 MLs of graphene, because the corresponding curves show two pronounced minima. From the darker areas labeled G, the curve shows three minima, so these areas have 3 MLs of graphene. Because most of the surface on this sample has a graphene coverage of $2 \mathrm{MLs}$, we henceforth refer to this sample as the 2-ML sample. C 1s spectra similar to those shown in Fig. 2(a) were collected from this sample. The only major difference compared to the Fig. 2(a) spectra was that the extracted $\mathrm{G} / \mathrm{SiC}$ peak intensity ratio of 6.3 (at $600 \mathrm{eV}$ ) was, as expected, lower, reflecting the lower graphene coverage on this sample. 


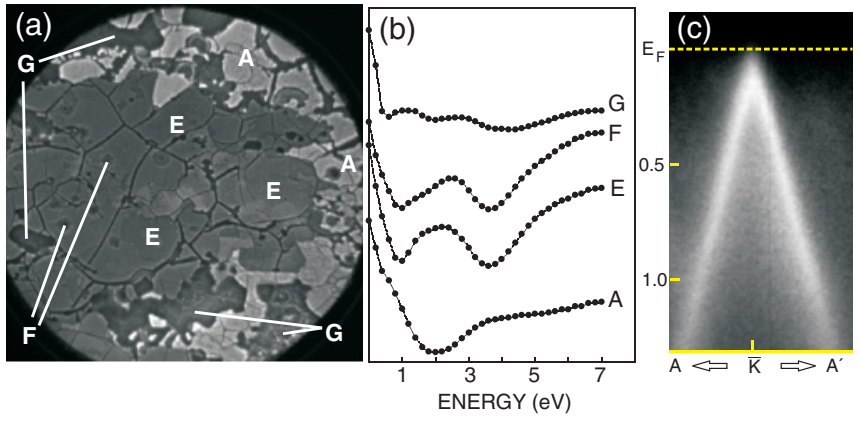

FIG. 3. (Color online) (a) LEEM image recorded from a second sample at $0.4 \mathrm{eV}$ and a field of view of $25 \mu \mathrm{m}$. (b) Electron reflectivity curves extracted from the areas labeled A and E-G. (c) The $\pi$-band recorded at the K-point from this sample with a graphene coverage of mainly 2 MLs. A photon energy of $33 \mathrm{eV}$ was used. This second sample was grown at $1800{ }^{\circ} \mathrm{C}$ for $20 \mathrm{~min}$ in an Ar pressure of 500 mbar.

The $\pi$-band recorded from this 2-ML graphene sample around the K-point using a photon energy of $33 \mathrm{eV}$ is shown in Fig. 3(c). As in Fig. 2(b), the Dirac point is located fairly close to the Fermi level, within $75 \mathrm{meV}$ in this case, rather than at $215 \mathrm{meV}$ below the Fermi level ${ }^{11}$ as previously determined. In addition, because the graphene grain size for this sample was only on the order of few hundred nanometers, a few $\pi$-cones were always detectable when moving the light spot over the sample. However, the Dirac point of the different observed cones was always located at the same energy position. If larger grain sizes could be obtained, it should be possible to find areas on the surface were only one set of $\pi$-cones was detected, as demonstrated later for a sample with a larger number of graphene layers.

A LEEM image collected from a third sample at a voltage of $1.3 \mathrm{eV}$ and a field of view of $10 \mu \mathrm{m}$ is shown in Fig. 4(a). This is the sample grown on a $4 \mathrm{H}-\mathrm{SiC}$ substrate. Extracted reflectivity curves from areas 1-8 are plotted in Fig. 4(b). Although clear domains appear in the LEEM image, the reflectivity curves show that several of them have the same number of graphene layers. Six minima are clearly observed in curves 1,2 , and 6-8, whereas curves 3-5 at first appear to have only five minima. However, when looking in detail at these latter three curves, there appears to be a weak minimum at $\sim 6.5 \mathrm{eV}$ in these curves as well. The coverage may thus actually be 6 MLs over the whole area in Fig. 4(a). Regardless, whether the whole surface has 6-ML coverage or some areas have 5 MLs is not important for the message we want to convey from this sample. The main message is contained in Fig. 4(c) and 4(d). Selected-area LEED patterns collected using a probing area of $400 \mathrm{~nm}$ from positions 1 and 2 in Fig. 4(a) are shown in the two upper panels in Fig. 4(c). It is obvious from these LEED patterns that a grain size $>400 \mathrm{~nm}$ has been accomplished on this sample and that the six graphene layers are stacked so that adjacent layers are not rotated with respect to each other. The bottom panel in Fig. 4(c) shows the LEED pattern collected using a $5-\mu \mathrm{m}$ probing area at position 2 . The sampled area then includes domains 1 and 2 . It is obvious that two grains of different azimuthal orientations are now probed, because this LEED pattern simply represents a superposition of the patterns shown in the upper two panels. To further emphasize
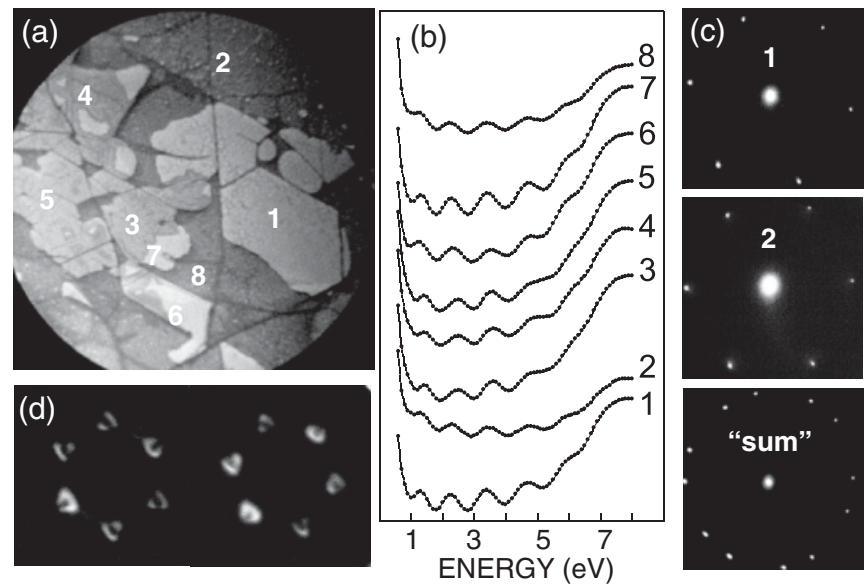

FIG. 4. (a) LEEM image recorded from a third sample at $1.3 \mathrm{eV}$ and a field of view of $10 \mu \mathrm{m}$. This is the sample grown on a $4 \mathrm{H}-\mathrm{SiC}$ substrate. (b) Electron reflectivity curves extracted from areas 1-8. (c) The two upper panels show selected-area LEED patterns collected at $45 \mathrm{eV}$ using a probing area of $400 \mathrm{~nm}$ from positions 1 and 2 in (a). The lower panel shows the LEED pattern collected using a 5- $\mu \mathrm{m}$ aperture at position 2. (d) Two constant energy photoelectron angular distribution patterns $\left(E_{i}, k_{x}, k_{y}\right)$ of the $\pi$-band collected using a probing area of $800 \mathrm{~nm}$ and a photon energy of $45 \mathrm{eV}$ at an energy of $\sim 2 \mathrm{eV}$ below the Fermi. One Dirac cone centered on each of the six $\mathrm{K}$-points in the Brillouin zone is clearly observed. This third sample was grown at $1900{ }^{\circ} \mathrm{C}$ for $15 \mathrm{~min}$ in an Ar pressure of $850 \mathrm{mbar}$.

that grains of six layers of graphene can form on the C-face and be stacked in such a way that no rotational disorder exists between adjacent layers, some constant energy photoelectron angular distribution patterns $\left(E_{i}, k_{x}, k_{y}\right)$ of the $\pi$-band ${ }^{20}$ were also recorded. Two such patterns collected using a probing area of $800 \mathrm{~nm}$ and a photon energy of $45 \mathrm{eV}$ at an energy of $\sim 2 \mathrm{eV}$ below the Fermi energy are shown in Fig. 4(d). Only six Dirac cones, which appear triangular at this initial energy, ${ }^{20}$ centered on the six K-points in the Brillouin zone are observed in these patterns. They were collected at two different locations outside the area shown in Fig. 4(a), but in both locations there were six graphene layers. These patterns show clearly that well-ordered grains of multilayer graphene larger than $800 \mathrm{~nm}$ have formed and that adjacent graphene layers are not rotated relative to each other. At this photon energy, separate and distinguishable signals from three to four layers of graphene have been demonstrated from Si-face samples. ${ }^{12,19}$ Moreover, the photoelectron angular distribution pattern recorded ${ }^{20}$ from bilayer graphene shows two $\pi$-cones of fairly similar intensity centered on each K-point, so any rotational disorder present between the two and the three uppermost graphene layers would show up directly in these patterns. The two patterns, in Fig. 4(d), show that the different grains probed have different azimuthal orientations. When selecting a probing area that included, e.g., two grains of different azimuthal orientation, two sets of superimposed Dirac cones were detectable, similar to the two superimposed micro-LEED patterns shown in the bottom panel in Fig. 4(c). The constant energy photoelectron angular distribution patterns thus show the same result as the selected area LEED patterns and provide additional proof of the formation of ordered grains of graphene on the $\mathrm{C}$-face, 


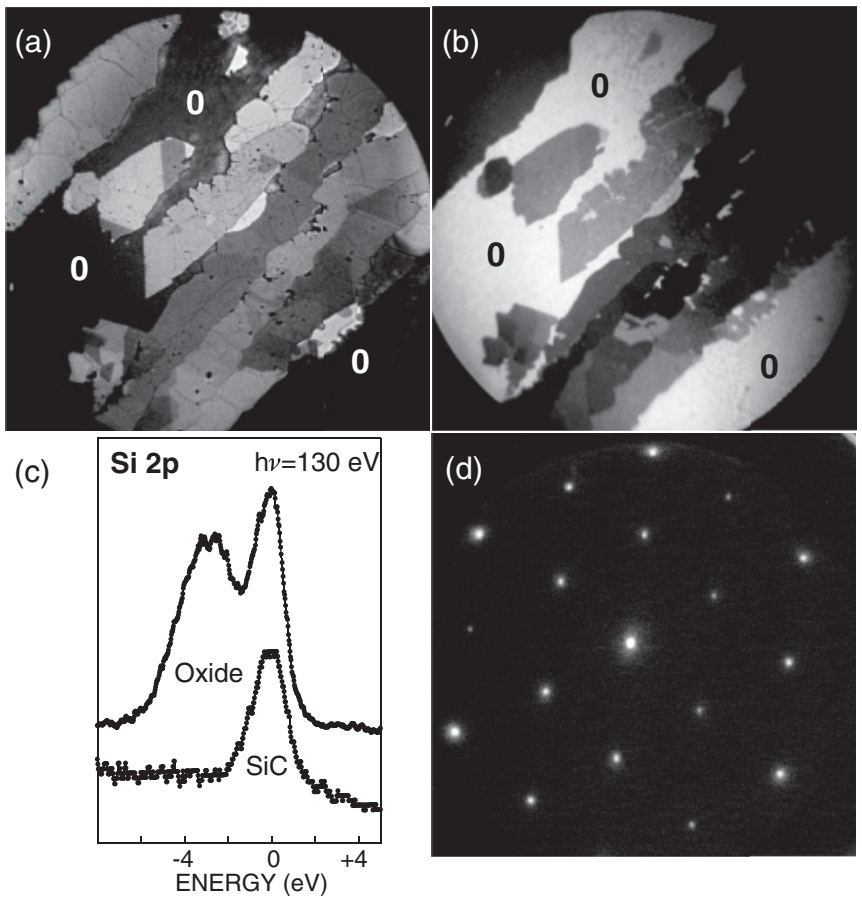

FIG. 5. (a) LEEM image collected from a fourth sample at a voltage of $1.3 \mathrm{eV}$ and a field of view of $50 \mu \mathrm{m}$. (b) XPEEM image collected from the same area at an energy of $1.4 \mathrm{eV}$ using $130 \mathrm{eV}$ photons, showing the variation in work function over the surface. The bright areas labeled 0 (which are the same as the dark gray areas labeled 0 in (a) correspond to regions where no graphene has formed. The gray areas show where graphene has formed, and the difference in darkness indicates that the number of graphene layers is different. (c) The upper curve shows the Si $2 p$ spectrum collected from the bright areas in (b); the bottom curve shows the Si 2p spectrum obtained from the gray graphene-covered areas. (d) Selected-area LEED pattern collected from the bright areas, at $45 \mathrm{eV}$, showing a silicate $\sqrt{3} \times \sqrt{3} \mathrm{R} 30^{\circ}$ diffraction pattern. This fourth sample was grown at $2000{ }^{\circ} \mathrm{C}$ for $10 \mathrm{~min}$ in an Ar pressure of 850 mbar.

with no rotational disorder between adjacent layers. However, not so homogenous areas of graphene are in general observed on the $\mathrm{C}$-face, so the number of graphene layers is within \pm 1 ML from the nominal value specified. Later, examples of this from two other samples are given.

The LEEM image in Fig. 5(a), collected from a fourth sample at a voltage of $0.7 \mathrm{eV}$ and a field of view of $50 \mu \mathrm{m}$, shows areas where different numbers of graphene layers have formed but also areas where no graphene has formed. The XPEEM image in Fig. 5(b), collected from the same area, illustrates this in a direct way. That image was collected at $1.4 \mathrm{eV}$ using $130 \mathrm{eV}$ photons and thus shows the variation in work function over the surface. When selecting the energy so that the intensity of the $\mathrm{Si} 2 \mathrm{p}$ photoelectron peak from the $\mathrm{SiC}$ substrate was instead detected, an almost identical image was obtained. Thus, the bright areas labeled 0 in Fig. 5(b), which are the same as the dark gray areas labeled 0 in Fig. 5(a), correspond to regions on the surface where no graphene has formed. The gray areas, on the other hand, show where graphene has formed, and the difference in darkness within these areas indicates that the number of graphene layers formed is different. Electron reflectivity curves extracted from these gray areas showed that the number of graphene layers varied from 3 to 8 MLs over these gray areas. To determine variations in the chemical composition over the surface, $\mathrm{Si}$ $2 \mathrm{p}$ and $\mathrm{C} 1 \mathrm{~s}$ spectra were collected using a probing area of $2 \mu \mathrm{m}$. The Si $2 \mathrm{p}$ spectrum obtained from the bright areas in Fig. 5(b) is shown by the upper curve in Fig. 5(c). The bottom Si 2 p spectrum in Fig. 5(c) shows the typical spectrum obtained from the gray areas and is interpreted to originate from the $\mathrm{Si} 2 \mathrm{p}$ level in the $\mathrm{SiC}$ substrate. The upper curve is seen to contain a component shifted almost $3 \mathrm{eV}$ to a larger binding energy, and it originates from an oxide formed on the $\mathrm{SiC}$ surface. ${ }^{21} \mathrm{C}$ 1s spectra recorded from the bright areas in Fig. 5(b) showed only the component from the SiC substrate, whereas from the gray areas, the graphene component was the dominant one when utilizing a photon energy of $450 \mathrm{eV}$. The micro-LEED pattern in Fig. 5(d) collected from the bright areas, at $60 \mathrm{eV}$, shows the $\sqrt{3} \times \sqrt{3} \mathrm{R} 30^{\circ}$ diffraction pattern expected from a $\mathrm{SiC}$ surface with an ordered silicate layer on. ${ }^{22}$ These findings are similar to the recently reported results $^{7}$ of graphene growth on the $\mathrm{SiC}(000-1)$ surface in an Ar environment. Our speculation is that in the atmospheric Ar environment utilized, the suppression of Si sublimation becomes so efficient for $\mathrm{C}$-face $\mathrm{SiC}$ that $\mathrm{Si}$-covered areas remain after the growth step and these areas then oxidize when exposing the surface to air. On surfaces like this, where large patches of oxide existed, we could observe effects of LEEM electron beam exposure. The spot/area exposed to the beam appeared darker in the LEEM image when an oxide/silicate layer was detectable. We therefore also tried to remove the oxide/silicate on the surface by hydrofluoric acid (HF) etching after growing the graphene but prior to measurements. The oxide patches, where no graphene had formed, naturally could not be eliminated by HF etching, but after etching we never observed effect of LEEM electron beam exposures on areas where graphene had formed. Moreover, we found that even on such nonhomogenous surfaces, the graphene formed on the different patches could contain fairly large well-ordered grains, as shown later.

The LEEM image in Fig. 6(a) was recorded from a fifth sample at $0.4 \mathrm{eV}$ and a field of view of $25 \mu \mathrm{m}$. This sample was etched in HF for $2 \mathrm{~min}$, thoroughly rinsed in deionized water, and dried before measurements. The extracted electron reflectivity curves, displayed in Fig. 6(b), show that in areas $1,2,3$, and 4, respectively, 3, 1, 4, and 2 MLs of graphene have formed. Before discussing what curves 5-8 show and correspond to, let us look at the quality of the graphene layers formed. The micro-LEED patterns shown in Fig. 6(c) were collected from areas 1-3 using a probing area of $1.5 \mu \mathrm{m}$. The patterns from the 3- and 4-ML areas (labeled 1 and 3 , respectively) show mainly a graphene $1 \times 1$ pattern. This demonstrates again that fairly large graphene grains can form that show no trace of rotational disorder between adjacent layers. On this surface, we could actually have used a probing area of $5 \mu \mathrm{m}$ and recorded a $1 \times 1$ LEED pattern from area 1 that had 3 MLs of graphene. In the diffraction pattern from the $1-\mathrm{ML}$ area (labeled 2 ), the $1 \times 1$ spots dominate but three additional weak spots around each $1 \times 1$ spot are also visible. These additional spots are most easily observed around the 0 -order spot and may have an origin similar to the additional 
$6 \sqrt{3} \times 6 \sqrt{3} \mathrm{R} 30^{\circ}$ spots observed on $\mathrm{Si}$-face $\mathrm{SiC}$ samples that originate from the carbon buffer layer forming on that surface. On $\mathrm{C}$-face $\mathrm{SiC}$, the graphene has been suggested to develop on either the $\mathrm{C}$-rich $2 \times 2$ or the $3 \times 3$ reconstructed surface, ${ }^{4,23}$ and our speculation is that these additional weak spots may be linked to the existence of some ordered aggregate of atoms at the graphene $\mathrm{SiC}$ interface. Some different and weaker superstructure spots were observed in the diffraction pattern from the 3-ML areas but not in the pattern from the 4-ML areas. However, before suggesting an explanation of these, further investigations are required. Let us now return to areas 5-8 on this surface. The corresponding curves in Fig. 6(b) show no pronounced minima, so it appears questionable whether there is any graphene in these areas. Therefore, we checked these areas using XPEEM and collected C $1 \mathrm{~s}$ and $\mathrm{Si} 2 \mathrm{p}$ spectra from some of them to determine the chemical composition.

The XPEEM image in Fig. 7(a) shows essentially the same area as the LEEM image in Fig. 6(a). This XPEEM image, collected at $0.1 \mathrm{eV}$ using $130 \mathrm{eV}$ photons, shows the variation in work function over the surface, and the different areas have been labeled in the same way as in Fig. 6(a). Again, bright areas correspond to regions where no graphene has formed, whereas the gray areas indicate graphene formation. To further confirm this, C $1 \mathrm{~s}$ spectra collected from areas 1 , 6 , and 2, using $450 \mathrm{eV}$ photons and a probing area of $1.5 \mu \mathrm{m}$, are displayed in Fig. 7(b). The G/SiC peak intensity ratio is seen to be fairly high in the spectrum from area 1, somewhat smaller from area 2, and around 1 from area 6. It appears that the gray pattern on area 6 originates from graphene and

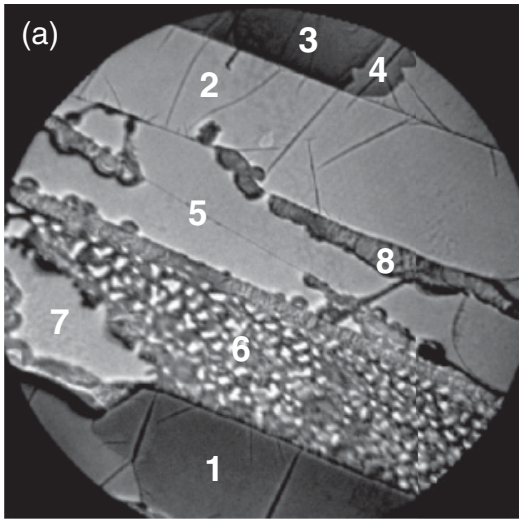

(c)
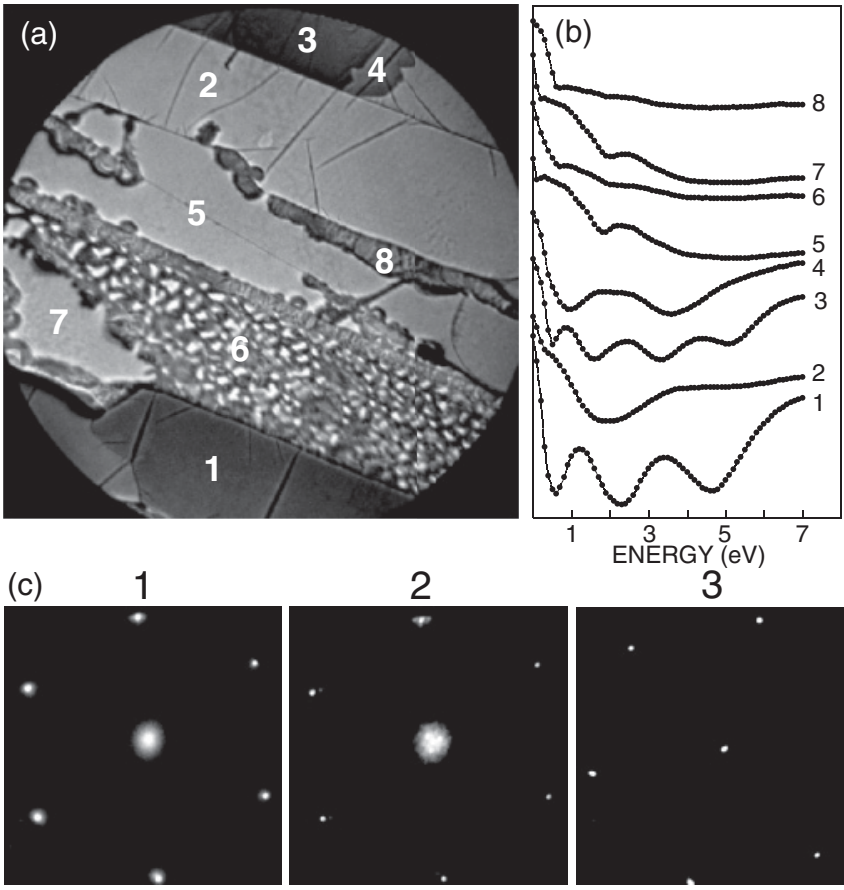

3

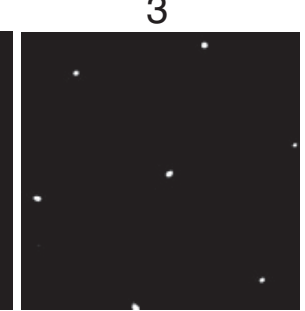

FIG. 6. (a) LEEM image recorded from a fifth sample at $0.4 \mathrm{eV}$ and a field of view of $25 \mu \mathrm{m}$. (b) Electron reflectivity curves extracted from areas 1-8. (c) Selected-area LEED pattern collected at $45 \mathrm{eV}$ from areas 1-3 using a probing area of $1.5 \mu \mathrm{m}$. This fifth sample was grown at $1900{ }^{\circ} \mathrm{C}$ for $15 \mathrm{~min}$ in an Ar pressure of $850 \mathrm{mbar}$. that area 6 contains a mixture of graphene and silicate. Also, area 8 contains graphene-but probably in small grains with different numbers of layers. No graphene component was detectable in $\mathrm{C}$ 1s spectra collected from areas 5 and 7. An oxide component was resolvable in the $\mathrm{Si} 2 \mathrm{p}$ spectrum from these areas, indicating that they are "silicate" regions like the areas labeled 0 in Fig. 5(a) and 5(b).

The results presented here from five graphene samples grown on $\mathrm{C}$-face $\mathrm{SiC}$ in an $\mathrm{Ar}$ environment show, like previously reported $\mathrm{C}$-face results, that fairly small domains with different numbers of graphene layers typically form on this surface. This is quite different from the growth of large, homogeneous, single-layer graphene samples shown to be possible on Si-face SiC. ${ }^{13-15,18-20}$ However, contrary to earlier findings and claims concerning graphene grown on $\mathrm{C}$-face $\mathrm{SiC}$, in situ or in a furnace and Ar environment, adjacent graphene layers do not show the rotational disorder previously reported. Only when a fairly large probing area is utilized are ringlike LEED patterns obtained similar to those previously reported..$^{2,4,5,7}$ When using smaller apertures, $1 \times 1$ LEED patterns are shown to result from multilayer graphene regions on the surface. Such $1 \times 1$ diffraction patterns are not observable if adjacent layers were rotationally disordered. Our LEED results thus demonstrate clearly that ordered grains of graphene that have no rotational disorder between adjacent layers form on $\mathrm{C}$-face $\mathrm{SiC}$ when grown in a furnace at high temperatures and in an Ar environment. Similar results are obtained from all five samples presented, although the grain size is shown to vary. For the first two samples, a grain size of $\sim 400 \mathrm{~nm}$ was obtained, whereas for the fifth sample, a grain size $>5 \mu \mathrm{m}$ was achieved. As further support for our claim of no rotational disorder between adjacent layers, constant energy photoelectron angular distribution patterns $\left(E_{i}, k_{x}, k_{y}\right)$ recorded from a sample with $6 \mathrm{MLs}$ of graphene are also presented. When selecting a probing area of $800 \mathrm{~nm}$ and an initial energy $\sim 2 \mathrm{eV}$ below Fermi, only the six Dirac cones, appearing triangular at this initial energy, centered on the six K-points in the Brillouin zone are observable in the photoelectron angular patterns. Thus, these patterns show clearly that well-ordered grains of graphene larger than $800 \mathrm{~nm}$ have formed. Thus, graphene grown on C-face SiC
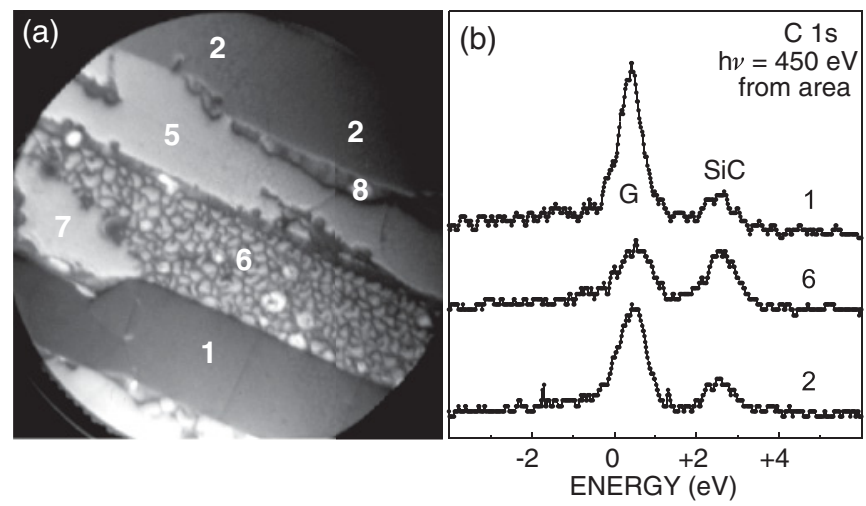

FIG. 7. (a) XPEEM image, collected from essentially the same area as in Fig. 6(a), at an energy of $0.1 \mathrm{eV}$ using $130 \mathrm{eV}$ photons. (b) $\mathrm{C} 1 \mathrm{~s}$ spectra recorded, using $450 \mathrm{eV}$ photons, from the areas labeled 1,6 , and 2 in (a). 
does not need a high density of rotational defects between layers, as previously reported. Many earlier studies utilized graphene grown in situ on $\mathrm{C}$-face $\mathrm{SiC}$. The graphene grain size is then expected to be considerably smaller, like that for graphene grown on $\mathrm{Si}$-face $\mathrm{SiC},{ }^{19}$ so a much smaller probing area than the smallest area of $400 \mathrm{~nm}$ that we utilized is probably required to distinguish among the different grains formed. In conventional LEED and in the first surface $\mathrm{X}$-ray diffraction study ${ }^{1}$ of graphene on $\mathrm{C}$-face $\mathrm{SiC}$, a much larger probing areas was utilized. In the latter case, it was specified to be $\sim 50 \times 50 \mu \mathrm{m}^{2}$; for a conventional LEED, the probing area is typically even larger. All five of our samples presented here should, if investigated using only those tools, appear to have a rotational disorder between adjacent layers. Not the formation of ordered grains of graphene where neighboring grains have different azimuthal orientation, as revealed by our results when using a smaller probing area. Because neighboring graphene grains are shown to have different azimuthal orientations, the boundaries between the grains must accommodate this change in azimuthal orientation. Therefore, we cannot exclude the existence of areas with a rotational disorder between adjacent layers and that these layers may be interleaved. On our five graphene samples it is obvious that these areas between the different grains constitute a small fraction of the total graphene surface area. However, when using a very local probe like STM, such grain boundary areas should be possible to locate if large-enough graphene grains formed on the surface. Therefore, we believe that the graphene grain size has previously been very small on $\mathrm{C}$-face $\mathrm{SiC}$ samples studied by STM. This is only a speculation, but we find it difficult to comprehend why ordered graphene grains, with a size of a few micrometers, have so far not been distinguished in STM studies of the C-face. We can only suggest that the higher growth temperature that we applied, compared to earlier efforts for C-face graphene, results in the formation of larger ordered grains of graphene, as previously revealed for Si-face graphene. ${ }^{13-15,18-20}$

The rotational disorder between adjacent layers of graphene grown on the $\mathrm{C}$-face has been suggested to explain why epitaxial graphene films, tens of layers thick, show single-layer electronic properties, ${ }^{2,5}$ i.e., show a single $\pi$-band cone with the Dirac point at the Fermi energy. A recent experimental study ${ }^{11}$ of the substrate-induced charge carrier density profile in C-face epitaxial graphene determined the Fermi level in the first three heavily doped graphene layers to be located at 360,215 , and $140 \mathrm{meV}$ above the Dirac point. Because we, by LEEM, could determine the number of layers actually grown on the C-face samples, we did ARPES studies of two samples that showed a dominant contribution from 2 and 3 MLs of graphene, respectively. The Dirac point was found to be located within 75 and $50 \mathrm{meV}$, respectively, from the Fermi level-not the 215 and $140 \mathrm{meV}$ determined previously from a 63-layer-thick graphene sample. Because the ARPES probing area was larger than the graphene grain size, more than one K-point and $\pi$-cone was typically probed. Nevertheless, the Dirac point was found to be located at the same energy position for the different $\pi$-cones. Our results thus indicate that the electron carrier concentration induced in the second and third graphene layers on the C-face is less than $\sim 4 \times 10^{11} \mathrm{~cm}^{-2}$, as estimated from a model ${ }^{24}$ assuming linear band dispersion near the Dirac point. This concentration is only $\sim 4 \%$ of the electron concentration typically obtained for the first layer of graphene on the $\mathrm{Si}$-face. ${ }^{14}$

Our experimental results show that there is no rotational disorder between adjacent layers of the multilayer epitaxial graphene (MEG) films grown on $\mathrm{C}$-face $\mathrm{SiC}$. The obvious question then is, Why do the graphene layers of the MEG film appear decoupled and behave nearly identically to electronically isolated graphene sheets, i.e., why does the MEG film exhibit a single $\pi$-cone with the Dirac point located close to the Fermi level? The effect of graphene stacking on the band structure of C-face MEG films has been the subject of active experimental and theoretical study (see Ref. 25 and references therein). It is commonly stated ${ }^{25}$ that $\mathrm{C}$-face graphene is known to have highly ordered non-Bernal (non-AB) stacking, where adjacent graphene planes have commensurate relative rotations that are not $60^{\circ}$. An average relative rotation of $\sim 30^{\circ}$ was claimed, but much smaller relative rotations were also found to occur with significant frequency. Our selected-area LEED and photoelectron angular distribution pattern results indicate that relative rotation angles very close to $0^{\circ}$ should dominate, suggesting $\mathrm{AA}, \mathrm{AB}$, or $\mathrm{ABC}$ stacking. $\mathrm{AB}$ and $\mathrm{ABC}$ stacking theory predicts ${ }^{26,27}$ hyperbolic $\pi$-band dispersions in the vicinity of the K-point. Dirac-type linear dispersion relations result for AA stacking in the vicinity of the K-point due to the sixfold symmetry of this configuration. Experimentally, a single $\pi$-band with linear dispersion is observed close to the K-point, suggesting AA stacking, even though multiple bands are predicted by theory. Serious inconsistencies exist between calculated and experimental band structures, as pointed out in the recent study ${ }^{25}$ of commensurately rotated graphene sheets. It was speculated that a fundamental process not included in current theories might be needed to resolve this issue.

\section{SUMMARY}

Graphene was grown on the C-face of nominally on-axis $\mathrm{SiC}$ substrates using high-temperature sublimation and a buffer inert gas. The thickness, morphology, and electronic structure of five graphene samples were investigated using LEEM, XPEEM, PES, and LEED. The graphene thickness was determined to vary from 1 or 2 to 6 or $7 \mathrm{MLs}$, depending on the specific growth conditions utilized. The formation of fairly large grains (crystallographic domains) of graphene exhibiting sharp $1 \times 1$ spots in micro-LEED was revealed. Adjacent grains were found to show different azimuthal orientations. Recorded macro-LEED patterns mimicked earlier published ${ }^{2,4,5,7}$ and schematically drawn, ${ }^{3}$ strongly modulated, diffraction ring LEED patterns, showing contribution from several grains of different azimuthal orientations. We collected selected area, constant initial energy, photoelectron, angular distribution patterns that showed the same result. When utilizing a small sampling area, one Dirac cone centered on each of the six Kpoints in the Brillouin zone was clearly resolved. When using a larger aperture, several Dirac cones from differently oriented grains were detected, giving rise to a ringlike appearance that resembled the ringlike diffraction pattern in macroLEED. Our experimental results thus clearly showed the existence of distinct graphene grains with different azimuthal orientations, rather than showing that adjacent graphene layers 
are rotationally disordered, as previously reported for C-face graphene. ${ }^{1-7}$ The graphene grain size was found to vary among the different samples prepared. For some samples, a probing area of $400 \mathrm{~nm}$ was needed to detect the grains, and on one sample, a probing area of $5 \mu \mathrm{m}$ was used to collect a $1 \times 1$ LEED pattern from a multilayer graphene grain.

Using ARPES, we determined the position of the Dirac point relative to the Fermi level for the two samples that have dominant graphene coverage of 2 and 3 MLs, respectively. The Dirac point was found to be located within 75 and $50 \mathrm{meV}$, respectively, from the Fermi level—not as far away as determined previously from a multilayer graphene sample. ${ }^{11}$ Because the ARPES probing area was larger than the graphene grain size on these two samples, more than one K-point and $\pi$-cone was typically probed, but the Dirac point was found to be located at the same energy position for the different $\pi$-cones. Our results indicate that the electron carrier concentration induced in the second and third graphene layers on the $\mathrm{C}$-face is less than $\sim 4 \times 10^{11} \mathrm{~cm}^{-2}$.
In agreement with recently reported results $^{7}$ of graphene growth on $\mathrm{C}$-face $\mathrm{SiC}$ in an Ar environment, we also observed formation of patches where no graphene formed on some samples. Using XPEEM, we revealed the presence of oxidized $\mathrm{Si}$ on the patches, and micro-LEED showed the $\sqrt{3} \times \sqrt{3}$ R30 diffraction pattern of ordered silicate. Our speculation is that in the atmospheric Ar environment utilized, the suppression of $\mathrm{Si}$ sublimation becomes so efficient for $\mathrm{C}$-face $\mathrm{SiC}$ that some $\mathrm{Si}$-covered areas remain after the growth and these areas then oxidize when the surface is exposed to air. Moreover, we found that even on such nonhomogenous surfaces, the graphene formed could contain fairly large, well-ordered, multilayer graphene grains.

\section{ACKNOWLEDGMENTS}

Support from the Swedish Energy Administration and the European Science Foundation, EuroGRAPHENE program, is gratefully acknowledged. *lejoh@ifm.liu.se

${ }^{1}$ J. Hass, R. Feng, J. E. Millan-Otoya, X. Li, M. Sprinkle, P. N. First, W. A. de Heer, E. H. Conrad, and C. Berger, Phys. Rev. B 75, 214109 (2007).

${ }^{2}$ J. Hass, F. Varchon, J. E. Millan-Otoya, M. Sprinkle, N. Sharma, W. A. de Heer, C. Berger, P. N. First, L. Magaud, and E. H. Conrad, Phys. Rev. Lett. 100, 125504 (2008).

${ }^{3}$ J. Hass, W. A. de Heer, and J. Conrad, J. Phys. Condens. Matter 20, 323202 (2008).

${ }^{4}$ K. V. Emtsev, F. Speck, Th. Seyller, L. Ley, and J. D. Riley, Phys. Rev. B 77, 155303 (2008).

${ }^{5}$ M. Sprinkle, D. Siegel, Y. Hu, J. Hicks, A. Tejeda, A. TalebIbrahimi, P. Le Fèvre, F. Bertran, S. Vizzini, H. Enriquez, S. Chiang, P. Soukiassian, C. Berger, W. A. de Heer, A. Lanzara, and E. H. Conrad, Phys. Rev. Lett. 103, 226803 (2009).

${ }^{6}$ D. L. Miller, K. D. Kubista, G. M. Rutter, M. Ruan, W. A. de Heer, P. N. First, and J. A. Stroscio, Phys. Rev. B 81, 125427 (2010).

${ }^{7}$ L. N. Srivastava, G. He, R. M. Feenstra, and P. J. Fisher, Phys. Rev. B 82, 235406 (2010).

${ }^{8}$ H. Le Roux, Acta Metall. 33, 309 (1985).

${ }^{9}$ L. I. Johansson, F. Owman, and P. Mårtensson, Phys. Rev. B 53, 13793 (1996).

${ }^{10}$ F. Varchon, R. Feng, J. Hass, X. Li, B. Ngoc Nguyen, C. Naud, P. Mallet, J.-Y. Veuillen, C. Berger, E. H. Conrad, and L. Magaud, Phys. Rev. Lett. 99, 126805 (2007).

${ }^{11}$ D. Sun, C. Divin, C. Berger, W. A. de Heer, P. N. First, and T. B. Norris, Phys. Rev. Lett. 104, 136802 (2010).

${ }^{12}$ T. Ohta, A. Bostwick, J. L. McChesney, T. Seyller, K. Horn, and E. Rotenberg, Phys. Rev. Lett. 98, 206802 (2007).

${ }^{13}$ C. Virojanadara, M. Syväjarvi, R. Yakimova, L. I. Johansson, A. A. Zakharov, and T. Balasubramanian, Phys. Rev. B 78, 245403 (2008).
${ }^{14}$ K. V. Emtsev, A. Bostwick, K. Horn, J. Jobst, G. L. Kellogg, L. Ley, J. L. McChesney, T. Ohta, S. A. Reshanov, J. Röhrl, E. Rotenberg, A. K. Schmid, D. Waldmann, H. B. Weber, and Th. Seyller, Nature Mater. 8, 203 (2009).

${ }^{15}$ R. M. Tromp and J. B. Hannon, Phys. Rev. Lett. 102, 106104 (2009).

${ }^{16}$ R. Yakimova, T. Yakimov, and M. Syväjärvi, Patent application PCT/SE2011/050328.

${ }^{17}$ H. Hibino, K. Kagashima, F. Maeda, M. Nagase, Y. Kobayashi, and H. Yamaguchi, Phys. Rev. B 77, 075413 (2008).

${ }^{18}$ C. Virojanadara, A. A. Zakharov, R. Yakimova, and L. I. Johansson, Surf. Sci. 604, L4 (2010).

${ }^{19}$ C. Virojanadara, R. Yakimova, A. A. Zakharov, and L. I. Johansson, J. Phys. D Appl. Phys. 43, 374010 (2010).

${ }^{20}$ A. A. Zakharov, C. Virojanadara, S. Watcharinyanon, R. Yakimova, and L. I. Johansson, IBM J. Res. Dev. 55(4), 6 (2011).

${ }^{21}$ C. Virojanadar and L. I. Johansson, Surf. Sci. 505, 358 (2002).

${ }^{22}$ J. Bernhardt, J. Schardt, U. Starke, and K. Heinz, Appl. Phys. Lett. 74, 1084 (1999).

${ }^{23}$ F. Hiebel, P. Mallet, F. Varchon, L. Magaud, and J.-Y. Veuillen, Phys. Rev. B 78, 153412 (2008).

${ }^{24}$ A. H. Castro Neto, F. Guinea, N. M. R. Peres, K. S. Novoselov, and A. K. Geim, Rev. Mod. Phys. 81, 109 (2009).

${ }^{25}$ J. Hicks, M. Sprinkle, K. Sheppard, F. Wang, A. Tejeda, A. Taleb-Ibrahimi, F. Bertran, P. Le Fèvre, W. A. de Heer, C. Berger, and E. H. Conrad, Phys. Rev. B 83, 205403 (2011).

${ }^{26}$ J. Borysiuk, J. Soltys, and J. Piechota, J. Appl. Phys. 109, 093523 (2011).

${ }^{27}$ C. L. Lu, C. P. Chang, Y. C. Huang, J. H. Ho, C. C. Hwang, and M. F. Lin, J. Phys. Soc. Jpn. 76, 024701 (2007). 